\title{
Analyst
}

CORRECTION

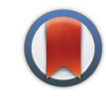

CrossMark

Cite this: Analyst, 2016, 141, 4804

DOI: 10.1039/c6an90038k

www.rsc.org/analyst

\section{Correction: Total viable bacterial count using a real time all-fibre spectroscopic system}

\author{
E. Bogomolny
}

Correction for 'Total viable bacterial count using a real time all-fibre spectroscopic system' by E. Bogomolny et al., Analyst, 2013, 138, 4112-4119.

S. Swift and F. Vanholsbeeck wish to resign their authorship of this paper as following further experiments in this area they are no longer confident that the conclusions, relating to the reported range for the detection of bacterial concentrations, are robust. E. Bogomolny remains in support of the reported conclusions as they stand.

The Royal Society of Chemistry apologises for these errors and any consequent inconvenience to authors and readers. 